\title{
Repetition priming mediated by task similarity in semantic classification
}

\author{
MAGGIE J. XIONG, JEFFERY J. FRANKS, and GORDON D. LOGAN \\ Vanderbilt University, Nashville, Tennessee
}

\begin{abstract}
In the present study, the specificity of repetition priming between semantic classificationtasks was examined using Osgood's (Osgood, Suci, \& Tannenbaum, 1957) semantic space as a heuristic for determining the similarity between classifications. The classification tasks involved judging the meaning of words on semantic scales, such as pleasant/unpleasant. The amount of priming across classifications was hypothesized to decrease with increasing distance (decreasing similarity) between semantic scales in connotative semantic space. The results showed maximum repetition priming when the study and the test classifications were the same, intermediate degrees of priming when the study and the test classification scales shared loadings on semantic factors, and little priming when the study and the test classification scales loaded primarily on orthogonal semantic factors - that is, when the distance between scales was maximized. Consistent with the transfer-appropriate processing framework, repetition priming in semantic classifications was highly task specific, decreasing with increasing distance between classification scales.
\end{abstract}

Repetition priming is a facilitation or a bias in performance on a stimulus that results from past experience with the stimulus. It manifests experimentally as faster reaction time (RT), improved accuracy, or bias toward one response over another (Jacoby \& Dallas, 1981; Jacoby \& Witherspoon, 1982; McAndrews \& Moscovitch, 1990; Ratcliff \& McKoon, 1996; for reviews, see Roediger \& McDermott, 1993; Schacter, 1987). Repetition priming happens independently of people's awareness or conscious control. Tests based on the repetition priming phenomenon, such as word or picture identification (Jacoby, Baker, \& Brooks, 1989; Jacoby \& Hayman, 1987) and word stem completion (Graf \& Schacter, 1985; Graf, Squire, \& Mandler, 1984; Shimamura \& Squire, 1984), have been used to investigate the relation between declarative and procedural, or explicit and implicit, memories.

The transfer-appropriate processing (TAP) principle is a conceptual rule for reasoning about and investigating memory phenomenaincluding repetition priming (Franks, Bilbrey, Lien, \& McNamara, 2000). Originally proposed as a principle for studying explicit memory retrieval (Bransford, Franks, Morris, \& Stein, 1979; Morris, Bransford, \& Franks, 1977), TAP claims that the specific processing of specific stimuli will facilitate later processing of the same stimuli to the extent that the earlier and the later events have overlapping processes. TAP differs from and complements the encoding specificity principle (Tulving, 1979; Tulving \& Thomson, 1973) in that it emphasizes the match of the dynamic processing on study and test stimuli, in contrast to representations of study and test stimuli per se.

Correspondence concerning this article should be addressed to M. J. Xiong, 301 Wilson Hall, Vanderbilt University, Nashville, TN 37209 (e-mail: maggie.xiong@vanderbilt.edu).
In the present study, the specificity of repetition priming - that is, the specificity of transfer between semantic classification tasks - was examined. From a strict TAP perspective, repetition priming should be highly task specific and sensitive to the similarity between study and test tasks. TAP is a methodological rule for reasoning about the relations between tasks. Whenever there is repetition priming, there must be overlapping cognitive processes between the study and the test tasks. However, TAP by itself does not make any specific predictions about the relations between tasks (Franks et al., 2000; Gorfein \& Bubka, 1997). A basic question in implicit memory is what structure of memory mediates repetition priming. It is possible to use the TAP principle to empirically map out the relations between various classification tasks and, thereby, to reveal the organization of the implicit memory space post hoc from experiment results. However, the range of possible classification tasks is unlimited, and random selection of tasks is very ineffective. Some methodological strategy concerning the relation between various classification tasks is needed. The semantic differential framework (Osgood, Suci, \& Tannenbaum, 1957) was adopted as the theoretical basis for deciding similarity relations among semantic classification tasks in the present work. The hypothesis is that repetition priming in semantic classifications will vary in relation to the degree of overlapping cognitive processes on the same dimensions of the semantic differential space.

Previous applications of TAP to memory phenomena tended to distinguish between perceptual and conceptual processes or between explicit and implicit memories in general (Roediger, 1990; Roediger, Weldon, \& Challis, 1989; Schacter, 1992). For example, it was known that conceptually driven tasks benefited most from generating the tar- 
get, whereas data-driven tasks were performed best when subjects read the targets (Blaxton, 1989, 1992; Jacoby, 1983). Dissociation among conceptual tests was regarded as a problem for the TAP approach (McDermott \& Roediger, 1996), although it was also noted that "a distinction between perceptual and conceptual processing is likely too rough and that more fine-grained distinctions are needed" (Roediger \& McDermott, 1993, p. 118).

Instead of associating repetition priming with general perceptual or conceptual processing, more recent studies have highlighted the specificity of transfer between different tasks (Franks et al., 2000; Gorfein \& Bubka, 1997; Thompson-Schill \& Gabrieli, 1999; Vriezen, Moscovitch, $\&$ Bellos, 1995). The experiments in these studies typically had a two-phase, cross-task design. Subjects performed one or two different tasks at the study phase. They then performed the same or a different task at the test phase, with half the items constant and the other half new for the task. Repetition priming was calculated as the difference in RT between old and new items at test. Even though the study and the test tasks might be different, the facilitation, if any, was considered repetition priming because the stimuli were held constant. Maximum priming was observed when the study and the test involved the same task. When the study and the test tasks differed, there was either some or no transfer, depending on the specific tasks involved.

Like the present study, Vriezen et al. (1995) and Thompson-Schill and Gabrieli (1999) assumed that repetition priming would reflect the similarity between semantic classification tasks. These studies differed from the present study in that they approached the similarity relation between tasks from a domain-specific knowledge perspective. They postulated that semantic information might be organized according to domain-specific attributes (e.g., structural/visual or functional). Classification tasks were considered similar if they accessed the same semantic attributes. Vriezen et al. found some transfer when size ("Is it larger than a breadbox?") and relative dimension ("Is it taller than it is wide?") classifications were crossed. No priming was detected between size and manmade classification tasks. Thompson-Schill and Gabrieli replicated the transfer between same-domain tasks. However, they also noticed small but consistent priming when the study and the test tasks accessed supposedly distinct semantic domains. They theorized that there might be both specific and nonspecific semantic systems or that domainspecific attributes might interact. These different conclusions regarding cross-domain priming might have resulted from a methodological difference (Thompson-Schill and Gabrieli mixed, instead of blocked, different classifications during study). Or they might suggest that domainspecific knowledge by itself is not sufficient for understanding the relations between semantic classification tasks or the structure of implicit memory space.

On the other hand, Vaidya et al. (1997, Experiment 7) found statistically equivalent amounts of priming in an abstract/concrete classification task following either the same or a shallow study task (deciding whether the word appeared in upper- or lowercase). According to Vaidya et al., the abstract/concrete classification test is characterized by noncompetitive access of conceptual knowledge - that is, the direct access of one particular entry in conceptual knowledge-as opposed to competitive access, where multiple entries were accessed upon presentation of a cue, as in category-cued generation. When the memory test requires noncompetitive access, any encoding task that elicits the retrieval of the word during study may lead to full priming. If this is true, repetition priming in semantic classification tasks should be the same regardless of the similarity between study and test classifications.

With a unique way of determining the similarity relation among semantic classifications, the present work provided an alternative way to study the implicit memory space, as well as convergent evidence that repetition priming across semantic classifications is sensitive to the similarity between study and test classifications. The semantic differential is a framework for measuring the connotative meaning of words in semantic space. The space is defined by a set of orthogonal semantic dimensions. A word or concept in semantic space is similar to a point in Euclidean space, having projections to all the dimensions. To identify the major dimensions of semantic space, Osgood et al. (1957) had subjects rate words on a set of semantic scales (polar terms, such as good/bad and large/small). They then used factor analysis on correlations between these scales to isolate major semantic factors (i.e., dimensions). The first three most prominent factors were identified as the evaluation, potency, and activity factors in order of the proportion of variance accounted for. Typical scales reflecting the evaluation dimension were good/bad, kind/cruel, and ferocious/peaceful. Typical scales of the potency dimension were large/small and strong/weak. Fast/slow and sharp/dull were representative of the activity dimension. Additional factors were extracted in other studies, but the evaluation, potency, and activity factors remained the most prominent (Bentler \& LaVoie, 1972; Tzeng, 1975; Tzeng \& May, 1975; Wickens \& Lindberg, 1975).

From the perspective of the semantic differential framework, the meaning of a word varies multidimensionally in the semantic space. Something judged good may also be judged strong (e.g., hero). If the scales used for classification tasks load heavily on orthogonal dimensions, they are assumed to be independent of each other. Being good is independent of being strong, and vice versa. In relation to the TAP principle, it is assumed that there is little or no overlap in cognitive processing between the good/bad and the strong/weak classifications. In contrast, if classification scales have significant projections to the same semantic dimension(s), there is assumed to be overlapping processes between them. For instance, large/small and hard/soft both have significant projections to the potency dimension (Osgood et al., 1957). Symmetrical repetition priming was demonstrated between big/small and hard/ soft classifications by Franks et al. (2000).

In the present study, it was presumed that the similarity between semantic classifications can be determined by the extent of overlapping cognitive processes on the same se- 
mantic dimensions. To make the similarities more amenable to manipulation, the psychological distance between different classifications was calculated. According to Osgood et al. (1957) and the geometric model of similarity (Shepard, 1980, 1987), the similarity between two stimuli is inversely related to the distance between their representations in the multidimensionalpsychological space. By extending this assumption to the psychological task space, similarity between classifications can be manipulated by selecting scales with varying distances in the semantic space. The prediction was that the amount of repetition priming should change as a negative function of the distance between scales.

Eighteen experiments were conducted in the present study. Experiments 1-17 used the same cross-task design as that in Franks et al. (2000). A general method for these experiments is reported because they employed the same basic design and procedure. The results from Experiment 1-17 were analyzed together, too. Consistent with the hypothesis, maximum repetition priming happened when the study and the test classification tasks were the same, intermediate degrees of priming were found with an intermediate distance between classification scales, and little priming was observed when the study and the test classification scales fell on orthogonal dimensions - that is, when the distance between the scales was maximized. Experiment 18 showed that the relation between semantic distance and repetition priming is not an artifact of response mapping. This experiment is reported separately.

\section{EXPERIMENTS 1-17}

\section{General Method}

Subjects. Four hundred thirty-two subjects participated in Experiments $1-17$. They were students in undergraduate psychology courses at Vanderbilt University, who participated in the study for course credit. They had normal or corrected-to-normal vision. All the subjects signed a consent form to participate in the study.

Design. The transfer pattern between two semantic classification tasks was studied in a series of experiments. The two classifications could be either the same or different from each other, which constituted the same- and the cross-transfer conditions. A basic experimental design (Design A) and two variations (Designs B and C) were used in the study. Design A was a 2 (test classification) $\times 2$ (item type) within-subjects design, as is illustrated in Figure 1. The subjects contributed to both same- and cross-transfer conditions.

Because of the particular pairing of classifications across experiments in this study, some of the same-transfer conditions could have been performed multiple times if only Design A had been used. Designs B (Experiment 9) and C (Experiments 10-17) consequently were employed to reduce redundancy. They focused on cross-transfer conditions only. In Design B, the subjects performed two test classifications, both of which differed from the study classification. In Design C, the subjects performed a classification task that differed from the study classification for two successive blocks. The results were analyzed according to transfer conditions, instead of experiments.

Tasks. Six semantic scales, represented by bipolar adjective word pairs, such as pleasant/unpleasant and strong/weak, were used for the classification tasks. For instance, in strong/weak classification, the subjects decided whether an item referred to something strong or weak. Scales were selected according to a comprehensive consideration of three study results in Osgood et al. (1957, Analyses I and II and the thesaurus study). ${ }^{1}$ Table 1 contains the relevant data from Osgood et al., Analysis I. Pleasant/unpleasant and valuable/ worthless loaded mainly on the evaluation factor. Strong/weak loaded mainly on the potency factor. Fast/slow loaded exclusively on the activity factor. Two scales were chosen to be intermediate, in which case they loaded on at least two orthogonal factors: The active/ passive pair loaded on both potency and activity factors, and the young/old pair loaded evenly on all three semantic factors.

There were three major groups of transfer conditions, in accordance with the distance between study and test classification scales. Repeating scales (same-transfer conditions) for study and test classifications constituted the group of minimum distance conditions. There were six same-transfer conditions for six semantic scales. Maximum distance between scales was achieved by crossing scales loaded heavily and relatively exclusively on orthogonal factors for study and test classifications: six binary combinations of pleasant/ unpleasant, strong/weak, and fast/slow, with transfer direction (the order of study and test classifications) taken into account. The intermediate group consisted of pairs of scales loaded on common factor(s): pleasant/unpleasant and valuable/worthless, strong/weak and active/passive, fast/slow and active/passive, pleasant/unpleasant and young/old, and fast/slow and young/old. There were 10 intermediate conditions when transfer direction was taken into account. All together, there were 22 unique transfer conditions in the study.

The distance between scale $i$ and scale $j$ was calculated, using their loadings $(l)$ on the four factors $\left(f_{1-4}\right)$ in Table 1 , with the distance formula, $D_{i j}=\left[\Sigma\left(l_{f i}-l_{f j}\right)^{2}\right]^{1 / 2}$. The distance between repeating scales was presumed to be zero. $D_{i j}$ could assume any value between zero and one. The value of $D_{i j}$ ranged from zero to .93 in the present study, as is presented in Table 2 .

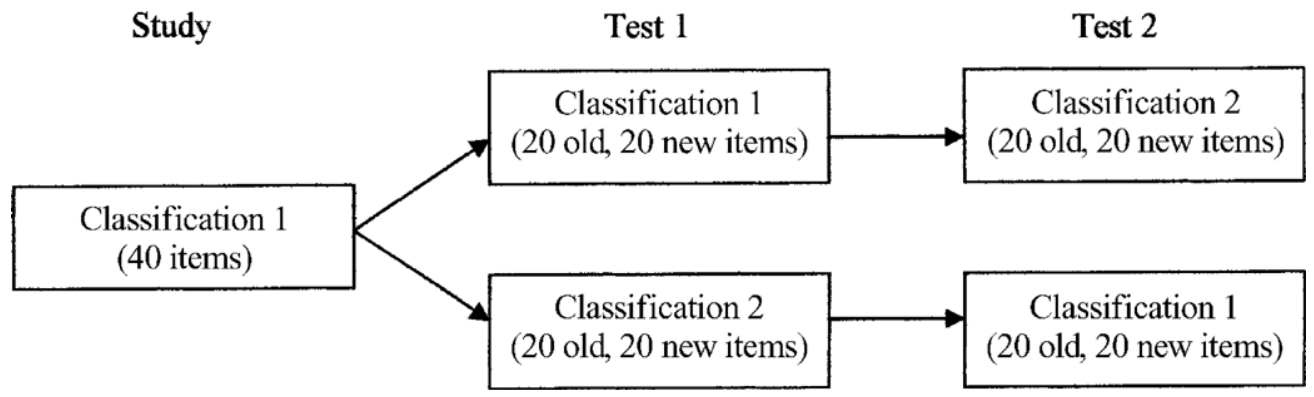

Figure 1. Basic experimental design (Design A). 
Table 1

Scales Used and Their Loadings on the Semantic Factors

\begin{tabular}{llcccc}
\hline & \multicolumn{5}{c}{ Semantic Factors } \\
\cline { 2 - 6 } \multicolumn{1}{c}{ Scale } & I & II & III & IV & $h^{2}$ \\
\hline Pleasant/unpleasant & .82 & -.05 & .28 & -.12 & .77 \\
Valuable/worthless & .79 & .04 & .13 & .00 & .64 \\
Strong/weak & .19 & .62 & .20 & -.03 & .46 \\
Active/passive* & .14 & .04 & .59 & -.02 & .37 \\
Fast/slow & .01 & .00 & .70 & -.12 & .50 \\
Young/old & .31 & -.30 & .32 & .01 & .29 \\
\% of total variance & 34 & 8 & 6 & 2 & 49 \\
\% of common variance & 69 & 15 & 13 & 3 & 100 \\
\hline
\end{tabular}

Note-Adapted from Osgood et al. (1957), Analysis I. Factors I, II, and III correspond to the evaluative, potency, and activity factors. *Active/ passive was considered an intermediate scale because it was highly loaded on both potency and activity factors in Analysis II and the thesaurus study.

A total of 26 same- and cross-transfer conditions were actually tested in this study, which are listed in Table 3. There were four replications: strong/weak-strong/weak (twice), valuable/worthlesspleasant/unpleasant, and young/old-pleasant/unpleasant .

Items. Experimental items were selected from a set of 156 words, which had a mean frequency of 170 per million $(S D=162$ per million; Kučera \& Francis, 1967). The length of words varied between 3 and 11 characters. In each experiment, there were 80 experimental items and 24 practice items (16 practice items for the Design C experiments), which were evenly divided into four subsets. For example, if the transfer condition under study was pleasant/unpleasantstrong/weak or strong/weak-pleasant/unpleasant, the study and the test lists were of equal numbers of items that could be judged as pleasant and strong,pleasant and weak, unpleasant and strong, and unpleasant and weak. This was achieved by selecting words according to their scores on relevant semantic factors (Heise, 1965) whenever possible - that is, when the scales under study were orthogonal to each other. In this example, the relevant factors were the evaluation and the potency factors, given that pleasant/unpleasant and strong/weak loaded exclusively on the evaluation and the potency factors, respectively. Equal numbers of words with positive scores and negative scores on the evaluation or the potency factor were selected. Positive scores were associated with pleasant or strong; negative scores were associated with unpleasant or weak. These subsets of items were selected to have the same mean scores on the relevant semantic factors, to equate word-specific influences on repetition priming between classification tasks. For example, the word steel scored 4.60 , whereas lake scored only -0.22 , on the potency factor. It could be much easier/faster for subjects to classify steel than lake on the strong/weak scale. Equalizing the average scores of the subsets controlled for such confounds. The same items were used for both transfer directions between a pair of tasks. Experimental items from the present study are presented in the Appendix for reference.

Procedure. There were two phases in the experiments. During the study phase, the subjects performed a classification task, such as pleasant/unpleasant, on 40 items. In the test phase of the Design A experiments (Experiments 1-6), the subjects performed the same classification task as that in the study phase, as well as a different classification. These classification tasks were blocked, with 20 old and 20 new items in each classification. The items from the study phase were divided into two complementary sets of 20 items each. They constituted the old items for the two test classifications. There was a total of 120 experimental trials. There were also 24 practice trials, 8 before each block. Repetition priming was defined as the difference in RT for new and old items at test-that is, RP $=\mathrm{RT}_{\text {new }}-$ $\mathrm{RT}_{\text {old }}$. The order of test classifications, old versus new word sets, and two complementary old word sets were counterbalanced. Presentation order of items was randomized for each subject. There was a 30 -sec break between blocks.

In the Design B experiment, the subjects performed two test classifications, both of which differed from the study classification. In the Design C experiments, the subjects performed a classification task that differed from the study classification for two successive blocks. There was no practice trial before the second block of the test classification. All other aspects of the Design B and C experiments were the same as those in Design A.

The subjects were tested individually. Task instructions and materials were presented on 8088 personal computers in 80-column lowercase font, in the default display color of green or orange. Each letter was about $2.5 \times 2.5 \mathrm{~mm}$ or $2.5 \times 5 \mathrm{~mm}$ (with extender) in size. The instructions included examples and stated that there was no right or wrong answer in the tasks. The subjects were asked to respond as soon as they had an impression of the meaning of the words with regard to the classification task, instead of spending a lot of time thinking about the classification. The subjects read the instructions and were then presented words one at a time for classification. They indicated different responses by pressing the " $\mathrm{Z}$ " or the "/?" key on the keyboard (e.g., "Z" for pleasant and "/?" for unpleasant classification). The dominant hand was assigned to positive classifications: pleasant, valuable, strong, active, fast, and young.

Each trial began with a ready signal $(*)$ that appeared in the middle of the screen for $500 \mathrm{msec}$. It was followed by the presentation of a word, which remained on the screen until the subject responded. The interval from the previous trial response to the next ready signal was $1,500 \mathrm{msec}$. The presentation order, response type, and RT were recorded for every experimental item.

\section{Results and Discussion}

Because there were no right or wrong answers in this study, all the trials were entered into the first analysis. An alpha level of .05 was used for all statistical tests. Repetition priming effects $\left(\mathrm{RT}_{\text {new }}-\mathrm{RT}_{\text {old }}\right)$ were analyzed with paired-sample $t$ tests for all transfer conditions. The results are reported in Table 3 . Three conditions were replicated in order to assess the reliability of the methods and results: strong/weak-strong/weak, valuable/worthlesspleasant/unpleasant, and young/old-pleasant/unpleasant. The results were reliable across replications, so the origi-

Table 2

Osgood Distance Between Semantic Scales Tested in the Present Study

\begin{tabular}{lllllll}
\hline \multicolumn{1}{c}{ Scale } & pu & vw & sw & ap & fs & yo \\
\hline Pleasant/unpleasant (pu) & .00 & .21 & .93 & & .91 & .58 \\
Valuable/worthless (vw) & & .00 & & & & \\
Strong/weak (sw) & & & .00 & .70 & .82 & \\
Active/passive (ap) & & & & .00 & .20 & \\
Fast/slow (fs) & & & & .00 & .58 \\
Young/old (yo) & & & & & .00 \\
\hline
\end{tabular}


Table 3

Reaction Times, Repetition Priming Effects, and Consistency Rates for Experiments 1-17

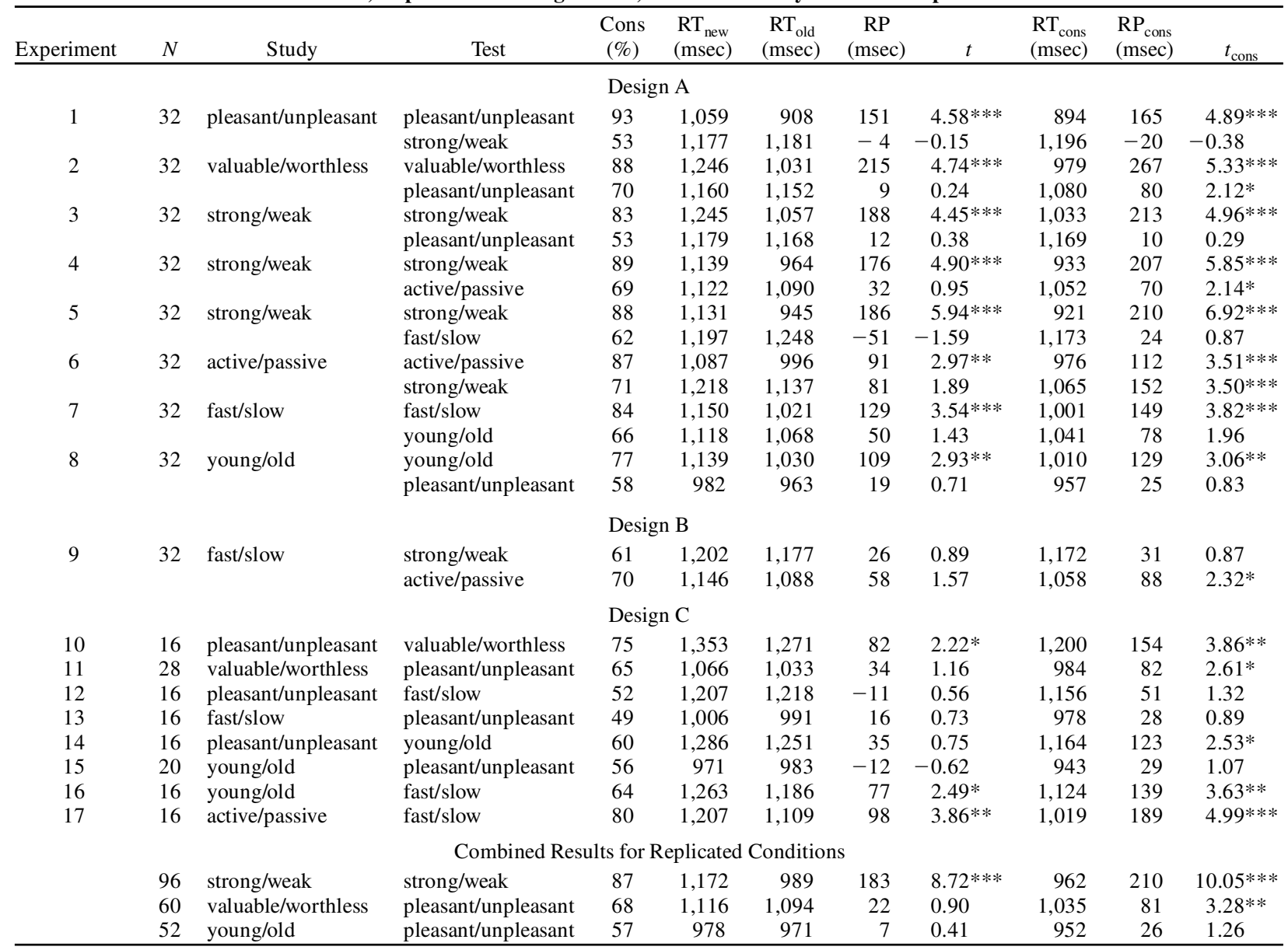

Note-Column "Cons" lists the percentage of consistent responses to old items at test in any given condition. Mean error can be calculated with $\mathrm{RP} / t$ or $\mathrm{RP}_{\text {cons }} / t_{\text {cons }} . \quad * p<.05$. **p $<.01 . * * * p<.001$.

nal conditions and replications were collapsed together in later analyses (see the bottom part of Table 3 ).

Mean repetition priming was calculated for the minimal, intermediate, and maximal distance task groups, collapsing across specific transfer conditions in each group. ${ }^{2}$ Maximum repetition priming occurred in the same-transfer (i.e., minimal distance) group ( $M=138 \mathrm{msec}, S E=13 \mathrm{msec}$. An intermediate level of repetition priming was found in the cross-transfer, intermediate distance group $(M=60 \mathrm{msec}$, $S E=11 \mathrm{msec}$ ). No repetition priming was detected in the cross-transfer, maximal distance group $(M=-1 \mathrm{msec}$, $S E=13 \mathrm{msec}$ ), even though the same stimuli were processed at study and test.

Figure 2 displays the repetition priming for all the transfer conditions. The transfer conditions are organized with increasing Osgood distance between study and test scales along the abscissa. The first six conditions are same-transfer conditions; the last six conditions are cross-transfer, maximal distance conditions, with scales representing orthogonal dimensions. Repetition priming varied in relation to the semantic differential distance between study and test scales. The correlation between repetition priming and Osgood distance was $-.80(p<.001)$.

Although priming effects between some pairs of tasks seemed to be asymmetrical, none of the comparisons was significant. For example, a relatively large asymmetry involved an 82-msec repetition priming effect from pleasant/ unpleasant study to valuable/worthless test, whereas repetition priming in the reverse direction was $22 \mathrm{msec}$. However, this difference was not statistically reliable $[t(74)=$ $1.23, p>.2]$.

In terms of the type of response-that is, whether an item should be rated positively or negatively on a scalethere was no correct answer in the present study. Response consistency was analyzed as an attempt to (1) check the validity of the responses and (2) examine the effect of stimulus-to-response mapping on RT and, more important, on repetition priming. If a subject gave the same hand response to the same item at test as in study, it was considered to be consistent whether the test classification dif- 


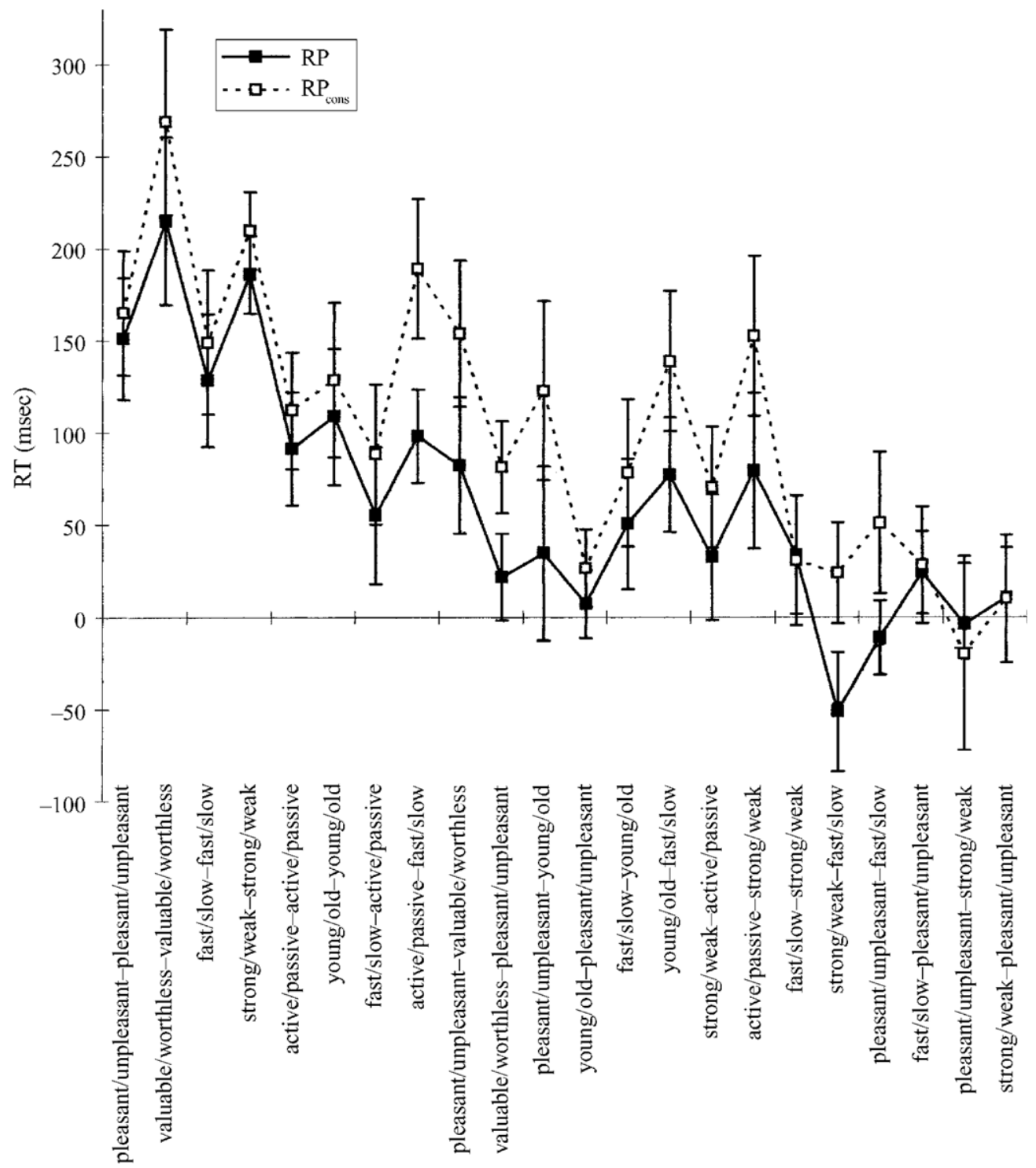

Transfer Conditions

Figure 2. Mean repetition priming (RP) and repetition priming for consistent responses $\left(\mathbf{R P}_{\text {cons }}\right)$. Error bars stand for $S E$ s. Transfer conditions are organized with increasing Osgood distance between study and test scales along the abscissa. The first six conditions belong to the same-transfer (i.e., minimal distance) group. The last six conditions belong to the cross-transfer, maximal distance group.

fered from the study classification or not. For example, when a subject made a dominant hand response to the item "flower" at pleasant-unpleasant study (indicating pleasant) and he/she again made a dominant hand response to "flower" in the strong-weak test classification (indicating strong), the test response was considered to be consistent. If he/she made a nondominant hand response in the strongweak test (indicating weak), the test response was counted as inconsistent.

The percentages of consistent responses for each condition are listed in Table 3 . The average consistency rate in the same-transfer conditions was $86 \%$, ranging from $77 \%$ to $93 \%$. Although RTs in the present study were longer than those in some other studies with semantic classification tasks (Franks et al., 2000; Vaidya et al., 1997; Vriezen et al., 1995; but see also Thompson-Schill \& Gabrieli, 1999), the moderately high consistency rates in the sametransfer conditions indicated that the validity of the responses in the present study was not compromised by some relatively unusual judgments. That the RT analysis with only consistent responses exhibited essentially the same pattern as the above analysis also suggested that RTs in the present study were long but valid (see below).

The consistency rates in cross-transfer conditions ranged from $49 \%$ to $80 \%$. Note that, simply on the basis of the methodologicalarrangement of test items into subsets, the 
expected consistency rate for cross-transfer conditions was $50 \%$ (see the Items section). The consistency rates between classifications representing orthogonal dimensions of the semantic space approximated the expected $50 \%$. However, responses for items in the intermediate distance group at test were biased toward the same (i.e., consistent) responses that were made during study. In fact, overall there was a large negative correlation between consistency rate and the Osgood distance between scales $(r=-.90$, $p<.001)$.

Given the negative correlation of consistency rate and Osgood distance between scales, if there were to be a benefit/cost in RT for making the sameldifferent motor response to the same item, this benefit/cost from stimulusto-response mapping could lead to more repetition priming in conditions with more consistent responses. Yet previous work by Logan (1990) has suggested otherwise. Logan examined the effects of consistent stimulus-toresponse and stimulus-to-interpretation mapping and found that only stimulus-to-interpretationmapping had an effect on priming. In the present study, RTs for consistent responses were faster than RTs for new items in the minimal and intermediate distance groups, but not in the maximal distance group (see Figure 3). This pattern reflected facilitation from consistency of interpretations, rather than of overt responses per se.

Furthermore, to equate any effect of stimulus-toresponse mapping across transfer conditions, the RTs for old items were recalculated using only consistent responses. Repetition priming was recalculated as $\mathrm{RP}_{\text {cons }}=$ $\mathrm{RT}_{\text {new }}-\mathrm{RT}_{\text {cons. }}$. The results are presented in Figure 2. For the same-transfer, minimal distance conditions and the cross-transfer, intermediate distance conditions, the size of the repetition priming is, in general, greater in this analysis than in the first analysis, which combined consistent and inconsistent responses. Priming effects in crosstransfer, maximal distance conditions remained nonsignificant. The correlation between $\mathrm{RP}_{\text {cons }}$ and Osgood

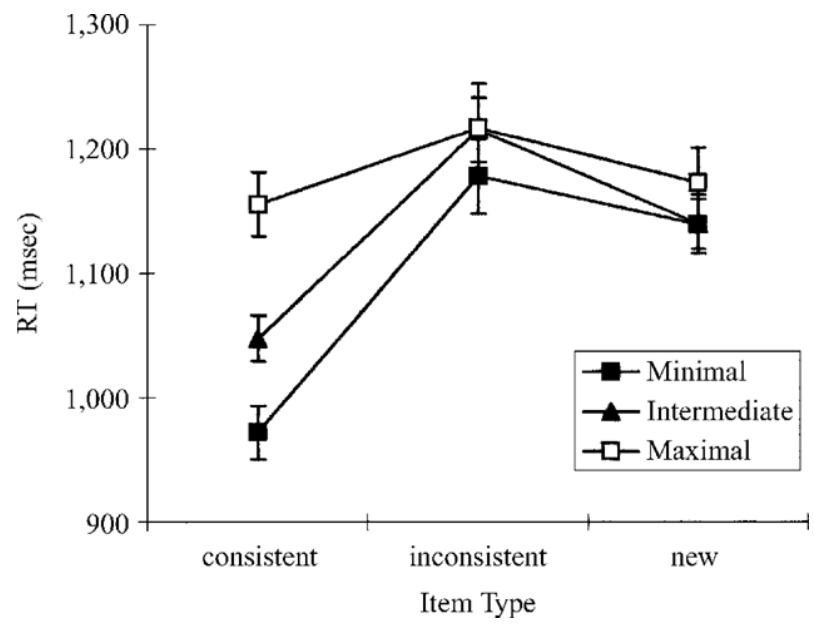

Figure 3. Mean reaction times for consistent and inconsistent responses to old items and to new items in the minimal, intermediate, and maximal distance groups. Error bars stand for $S E$ s. distance stayed high $(r=-.77, p<.001)$. The results from consistent responses agreed with the previous analysis. These results nicely support the hypothesis that similarity in connotative meaning between semantic scales, as measured by the semantic differential, reflects degree of overlap of processing in classification tasks and that this overlap in processing is directly related to the degree of repetition priming between tasks.

The correlation between consistency rate and semantic distance could be a manifestation of preexperimental covariation of item properties, which has no direct relation with transfer between classification tasks. ${ }^{3}$ For any stimulus, the properties being judged at study and test tend to covary when the scales are more similar to each otherthat is, when the semantic distance between scales is small. For example, the distance between fast/slow and activelpassive is smaller than that between fast/slow and pleasant/unpleasant. Something being fast is likely associated with its being active, but it does not have to be pleasant. This will lead to more consistent responses to old items when scales with smaller distances are crossed.

\section{EXPERIMENT 18}

The consistency analysis for Experiments 1-17 indicated that stimulus-to-response mapping did not affect transfer patterns between semantic classification tasks. However, all positive responses (pleasant, valuable, strong, active, fast, and young) were mapped to a subject's dominant hand in the above experiments. The effect of motor response mapping, if any, could not be addressed directly in those experiments. Experiment 18 was designed to investigate the possible influence of response mapping on repetition priming in semantic classifications. If reversing the response mapping between study and test classifications were to reduce or eliminate the priming effects, it would be evidence that the negative correlation between repetition priming and semantic distance resulted from the covariation of item properties. In contrast, if response mapping were to have no effect on priming effects, it would suggest that task transfer happened in relation to the semantic distance between classifications in the previous experiments.

Pleasant/unpleasant and valuable/worthless classifications were selected as the tasks for Experiment 18. This provided us with an extra chance to look at the possible asymmetrical transfer pattern between these classifications.

\section{Method}

Participants. Sixty-eight undergraduate students from the Vanderbilt University participated in the experiment for course credit. All the subjects signed a consent form for participation.

Items. There were 136 word items in the experiment, 96 experimental items and 40 practice items. Of the experimental items, 80 were from previous experiments on pleasant/unpleasant and valuable/ worthless classifications. Another 16 items were added to fill the present mixed factorial design, so that there would be 12 items in each cell. The mean frequency for experimental items was 141 per million (the word "bagel" has no corresponding entry in the norm; $S D=143$ per million; Kučera \& Francis, 1967). 
Procedure. Experiment 18 had a 2 (study classification) $\times 2$ (test classification) $\times 2$ (response mapping) $\times 2$ (item type) mixed factorial design. The subjects could be divided into two groups, depending on whether they had a pleasant/unpleasant or a valuable/ worthless classification at study. For half of the subjects from each group, the positive response (pleasant or valuable) was mapped to the dominant hand during study. For the other half of the subjects, the positive response was mapped to the nondominant hand. Fortyeight experimental trials were presented in a single block during study. All the subjects then performed both the same and the other classification at test. The test trials were presented in four blocks, two for each classification task. One of these two blocks had the same response mapping as the study phase; the other block had the reversed mapping. Twenty-four experimental trials were presented in every test block, half of which involved old items from the study phase and the other half of which consisted of new items. The order of the test classifications and response mapping was counterbalanced across subjects. Words were counterbalanced across test classifications and response mapping and were randomly assigned to old versus new word status. For both study and test blocks, there were eight practice trials at the beginning of each block and a 30 -sec break after each block.

The testing instructions and presentation sequences were the same as those described in Experiments 1-17. The presentation order, response type, and RT were recorded for experimental items.

\section{Results and Discussion}

With a more complicated design, Experiment 18 had fewer trials in each cell than did Experiments 1-17. To get more stable data, average RTs were calculated for each subject. Data from 4 subjects with extremely long RTs (more than two standard deviations away from the mean RT across all subjects) were replaced with data from another 4 subjects. There were 64 subjects in the analysis.

A mixed ANOVA was performed on the data. The means are listed in Table 4. No main effect of response mapping was observed. There was a significant main effect of item type $\left[F(1,62)=49.82, M S_{\mathrm{e}}=26,900.87, p<\right.$ $.001]$. RTs to old items were faster than RTs to new items in general. Unlike in previous experiments, there was a sign of task learning in Experiment 18, as manifested by the main effect of test classification $[F(1,62)=25.68$, $\left.M S_{\mathrm{e}}=36,422.45, p<.001\right]$. A test classification was performed more quickly if it was the same as the study classification.

No interaction involving response mapping was significant except for the interaction between study classification and response mapping $\left[F(1,62)=4.45, M S_{\mathrm{e}}=\right.$
$52,950.01, p<.05]$. The group of subjects who had the pleasant/unpleasant classification at study was slowed down by $40 \mathrm{msec}$ by the reversed response mapping at test. In contrast, the valuable/worthless group sped up by $44 \mathrm{msec}$ with the reversed mapping. This interaction most likely arose from the particular task/item combination in the present experiment.

There was an interaction between study and test classifications $\left[F(1,62)=12.21, M S_{\mathrm{e}}=36,422.45, p<.001\right]$. There was also a significant three-way interaction between study classification, test classification, and item type $\left[F(1,62)=4.09, M S_{\mathrm{e}}=30,387.91, p<.05\right]$. Visual inspection of the means suggested that these interactions were due to the fact that the valuable/worthless classification took longer and that it received more priming than did the pleasant/unpleasant classification at test, rather than being due to an asymmetrical cross-transfer pattern between pleasant/unpleasant and valuable/worthless classifications. For the pleasant/unpleasant test, the priming effect was $43 \mathrm{msec}$ less following the valuable/worthless classification (cross-transfer) than following the pleasant/ unpleasant classification (same-transfer), collapsing across response-mapping manipulations. For the valuablel worthless test, the difference between pleasant/unpleasant (cross-transfer) and valuable/worthless (same-transfer) study conditions was $38 \mathrm{msec}$. A planned comparison showed that these differences, 43 and $38 \mathrm{msec}$, were not statistically different from each other $[t(62)=0.09$, n.s. $]$. No asymmetry was found in the transfer pattern between pleasant/ unpleasant and valuable/worthless classifications. No other effects in this experiment were statistically reliable.

Overall, the results from Experiment 18 confirmed that stimulus-to-response mapping has no effect on the repetition priming pattern between semantic classification tasks. The transfer pattern between pleasant/unpleasant and valuable/worthless classifications is fairly symmetrical.

\section{GENERAL DISCUSSION}

In the present study, the similarity between study and test semantic classifications was manipulated by selecting classification scales with varying distance in the semantic space. Repetition priming at test decreased with increasing distance between study and test classification scales.

Table 4

Reaction Times and Repetition Priming Effects for Experiment 18 (in Milliseconds)

\begin{tabular}{|c|c|c|c|c|c|c|c|}
\hline \multirow[b]{2}{*}{ Study } & \multirow[b]{2}{*}{ Test } & \multicolumn{3}{|c|}{ Same Mapping } & \multicolumn{3}{|c|}{ Reversed Mapping } \\
\hline & & $\mathrm{RT}_{\text {new }}$ & $\mathrm{RT}_{\text {old }}$ & $\mathrm{RP}$ & $\mathrm{RT}_{\text {new }}$ & $\mathrm{RT}_{\text {old }}$ & $\mathrm{RP}$ \\
\hline \multirow{3}{*}{ Pleasant/unpleasant } & & Group & & & & & \\
\hline & pleasant/unpleasant & 972 & 891 & 80 & 1,051 & 946 & 105 \\
\hline & valuable/worthless & 1,168 & 1,035 & 133 & 1,165 & 1,070 & 96 \\
\hline \multirow{3}{*}{ Valuable/worthless } & & Group 2 & & & & & \\
\hline & valuable/worthless & 1,090 & 938 & 151 & 1,033 & 880 & 154 \\
\hline & pleasant/unpleasant & 1,043 & 1,011 & 32 & 1,030 & 963 & 67 \\
\hline
\end{tabular}


No reliable effect of response mapping on the repetition priming pattern between classifications was found.

For any particular semantic classification task, maximum repetition priming in that classification task was observed when the study task involved the same classification. This replicated previous findings by Franks et al. (2000) and Vriezen et al. (1995), which also showed maximum repetition priming in same-transfer conditions. Also noticeable in the present study was that although same-transfer conditions exhibited the most repetition priming as a group, the range of priming effects within this group was large, from 91 to $215 \mathrm{msec}$. The current distance hypothesis would seem to predict that priming effects in same-transfer conditions should be about the same, because the distance between study and test classification scales is the same-namely, zero distance. However, although the distance hypothesis was generally supported in the present study, other sources could affect the size of repetition priming effects as well.

Consider two such sources that could lead to the variability observed in same-transfer conditions. First, there might be a scaling effect. RTs differed from classification to classification in the present study. The longer it took to perform a classification task, the more repetition priming the items tended to receive during the test classification. In Experiments 1-17, the Pearson correlation between repetition priming and RT to new items in same-transfer conditions was .71 $(p=.12)$. The correlation is not significant, but there were only six observations in the correlation. Second, the self-similarity of the concepts (Barsalou, 1989, 1993) being judged (e.g., the concepts of pleasantness, value, strength, etc.) might have contributed to the variability in the same-transfer conditions. According to Barsalou, the self-similarity of concepts varies from concept to concept-some concepts have more stable representations than others. The present distance hypothesis is derived from a similarity hypothesis. It is totally compatible with the present hypothesis if repetition priming in same-transfer conditions varies with respect to the selfsimilarity of the concept being judged. We tentatively used the communality of a scale with the extracted semantic factors ( $h^{2}$ in Table 1) as an indicator for self-similarity, because it signals the common variance between a scale and a relatively stable semantic space. The correlation between repetition priming and $h^{2}$ for same-transfer conditions turned out to be $.60(p=.21)$. Although the correlation is not significant here, it is nevertheless a promising direction for future studies. A complete similarity model of repetition priming in semantic classifications should incorporate the self-similarity of semantic scales, as well as the similarity between different scales. But for the time being, the focus is on similarity between different scales.

The most striking result of the present study is that no reliable priming effect could be detected in maximal distance conditions - that is, when the classification tasks were characterized by processing on orthogonal semantic dimensions, even though the stimulus words were repeated.
This is in agreement with Vriezen et al. (1995), who reported that the benefit of repeated perceptual processes was negligible when study and test tasks tapped into different semantic domains. These results are in sharp contrast with the prediction of the noncompetitive response theory (Vaidya \& Gabrieli, 2000; Vaidya et al., 1997), which contends that as long as a word is encoded during study, there will be full priming if the test task requires a noncompetitive response. Although the noncompetitive response theory seemed to be supported by the finding that the abstract/concrete classification received equivalent amounts of priming following either the same or a perceptual task, similar experiments by Franks et al. (2000) have shown no priming in animacy or big/small classifications following a lexical decision task. Other, independent studies are needed to resolve the issue at this point.

When the study and the test classifications differed but involved processing on the same semantic dimension(s), there was a tendency for repetition priming to decrease with increasing distance between classification scales. Yet the correlation between priming effects in the intermediate group and Osgood distance was modest at best ( $r=$ -.26 , n.s.). We speculate that more up-to-date and largescale norm data of the semantic space are necessary when attempting to differentiate classification tasks within such a restricted range of similarities, especially when the effect of semantic distance may be obscured by variances introduced by the scaling effect and the self-similarity of concepts.

The present study demonstrated that, depending on the type of semantic classification subjects perform during study and test, repetition priming may or may not happen. Some studies (Light, Prull, \& Kennison, 2000; Vaidya et al., 1997) have reported that category verification, a task similar to semantic classification, does not respond to levelsof-processing manipulation. In their experiments, a conceptual task different from the category verification was used task during study. Since all conceptual tasks do not show full priming between them, results from levels-ofprocessing manipulationneed to be construed with caution.

Unlike Thompson-Schill and Gabrieli (1999), who showed small but consistent repetition priming between tasks accessing different domain-specific attributes, priming effects were unreliable between scales loaded mainly on orthogonal semantic factors. The present results provide no evidence for a nonspecific semantic system such as that described by Thompson-Schill and Gabrieli. In the semantic differential framework, similarities between scales are essentially represented by correlations between these scales. Extending this thinking to the case of functional and structural attributes, if a functional attribute is correlated with some structural/visual attribute, repetition priming may happen when tasks accessing these attributes are crossed, even if they supposedly are tapping into different semantic domains. As an example, repetition priming is very likely to happen between having wings and being able to fly classifications. 
There may be a general semantic system in the sense that the correspondence between all representations and their external referents is fine-tuned through similar mechanisms independently of modality or semantic domainfor example, by adjusting connection weights between the input and the output according to the same learning rules (McClelland, McNaughton, \& O'Reilly, 1995). In the present study, although dimensions of the semantic space are statistically orthogonal to each other, they presumably have developed in much the same way through associative learning and response generalization (Osgood et al., 1957). These dimensions do not have to represent different semantic systems. But the manner in which the underlying semantic system is accessed may be specific to the intentional acts called upon by different tasks, giving rise to task-specific repetition priming effects. This is the TAP view that repetition priming is a function of the relations between domain-specific processes. Specificity of transfer arises because processing related to the semantic dimensions is invoked differentially by different semantic classification tasks. Such a processing account is compatible with the component-of-processing view of repetition priming (Moscovitch, 1992; Vriezen et al., 1995; Witherspoon \& Moscovitch, 1989), which considers different brain areas as modules for performing different tasks. Transfer happens when there are overlapping component processes between the study and the test processing of the stimuli.

In conclusion, the semantic differential has been demonstrated to be a fruitful guiding heuristic for defining and manipulating semantic similarity. The findings suggest that, at least in part, repetition priming between study and test semantic classifications varies as a function of distance in the semantic differential space representing connotative meaning. In accordance with the TAP approach, specificity of transfer is suggested to be the result of differential processing, rather than of the accessing of distinct semantic systems.

\section{REFERENCES}

BARSALOU, L. W. (1989). Intraconcept similarity and its implications for interconcept similarity. In S. Vosniadou \& A. Ortony (Eds.), Similarity and analogical reasoning (pp. 76-121). Cambridge: Cambridge University Press.

BARSALOU, L. W. (1993). Flexibility, structure, and linguistic vagary in concepts: Manifestations of a compositional system of perceptual symbols. In A. F. Collins, S. E. Gathercole, M. A. Conway, \& P. E. Morris (Eds.), Theories of memory (pp. 29-101). Hillsdale, NJ: Erlbaum.

Bentler, P. M., \& La Voie, A. L. (1972). An extension of semantic space. Journal of Verbal Learning \& Verbal Behavior, 11, 174-182.

BLAXTON, T. A. (1989). Investigating dissociations among memory measures: Support for a transfer-appropriate processing framework. Journal of Experimental Psychology: Learning, Memory, \& Cognition, 15, 657-668.

Blaxton, T. A. (1992). Dissociations among memory measures in memory-impaired subjects: Evidence for a processing account of memory. Memory \& Cognition, 20, 549-562.

Bransford, J. D., Franks, J. J., Morris, C. D., \& Stein, B. S. (1979). Some general constraints on learning and memory research. In L. S. Cermak \& F. I. M. Craik (Eds.), Levels of processing in human memory (pp. 331-355). Hillsdale, NJ: Erlbaum.
Franks, J. J., Bilbrey, C. W., Lien, K. G., \& McNamara, T. P. (2000). Transfer appropriate processing (TAP) and repetition priming. Memory \& Cognition, 28, 1140-1151.

Gorfein, D. S., \& BUBKA, A. (1997). A transfer analysis of the repetition effect in the lexical and ambiguity decision tasks. Psychonomic Bulletin \& Review, 4, 232-236.

Graf, P., \& Schacter, D. L. (1985). Implicit and explicit memory for new associations in normal and amnesic subjects. Journal of Experimental Psychology: Learning, Memory, \& Cognition, 11, 501-518.

Graf, P., Squire, L. R., \& Mandler, G. (1984). The information that amnesic patients do not forget. Journal of Experimental Psychology: Human Learning \& Memory, 10, 164-178.

HeIsE, D. R. (1965). Semantic differential profiles for 1,000 most frequent English words. Psychological Monographs: General \& Applied, 79, 1-31.

JACовY, L. L. (1983). Remembering the data: Analyzing interactive processes in reading. Journal of Verbal Learning \& Verbal Behavior, 22, 485-508.

JACOBY, L. L., BAKeR, J. G., \& BRooKs, L. R. (1989). Episodic effects on picture identification: Implications for theories of concept learning and theories of memory. Journal of Experimental Psychology: Learning, Memory, \& Cognition, 15, 275-281.

JACOBY, L. L., \& DALlas, M. (1981). On the relation between autobiographical memory and perceptual learning. Journal of Experimental Psychology: General, 110, 306-340.

JACOBY, L. L., \& HAYMAN, C. A. (1987). Specific visual transfer in word identification. Journal of Experimental Psychology: Learning, Memory, \& Cognition, 13, 456-463.

JAcoby, L. L., \& Witherspoon, D. (1982). Remembering without awareness. Canadian Journal of Psychology, 36, 300-324.

KuČERA,H., \& Francis, W. N. (1967). Computationalanalysis of presentday American English. Providence, RI: Brown University Press.

Light, L. L., Prull, M. W., \& Kennison, R. F. (2000). Divided attention, aging, and priming in exemplar generation and category verification. Memory \& Cognition, 28, 856-872.

LogAN, G. D. (1990). Repetition priming and automaticity: Common underlying mechanisms? Cognitive Psychology, 22, 1-35.

McAndrews, M. P., \& Moscovitch, M. (1990). Transfer effects in implicit tests of memory. Journal of Experimental Psychology: Learning, Memory, \& Cognition, 16, 772-788.

McClelland, J. L., McNaughton, B. L., \& O’Reilly, R. C. (1995). Why there are complementary learning systems in the hippocampus and neocortex: Insights from the successes and failures of connectionist models of learning and memory. Psychological Review, 102, 419-457.

McDermott, K. B., \& Roediger, H. L., III (1996). Exact and conceptual repetition dissociate conceptual memory tests: Problems for transfer appropriate processing theory. Canadian Journal of Experimental Psychology, 50, 57-71.

Morris, C. D., Bransford, J. D., \& Franks, J. J. (1977). Levels of processing versus transfer appropriate processing. Journal of Verbal Learning \& Verbal Behavior, 16, 519-533.

Moscovitch, M. (1992). Memory and working-with-memory: A component process model based on modules and central systems. Journal of Cognitive Neuroscience, 4, 257-267.

Osgood, C. E., Suci, G. J., \& Tannenbaum, P. H. (1957). The measurement of meaning. Urbana: University of Illinois Press.

Ratcliff, R., \& McKoon, G. (1996). Bias effects in implicit memory tasks. Journal of Experimental Psychology: General, 125, 403-421.

Roediger, H. L., III (1990). Implicit memory: Retention without remembering. American Psychologist, 45, 1043-1056.

Roediger, H. L., III, \& McDermott, K. B. (1993). Implicit memory in normal human subjects. In H. Spinnler \& F. Boller (Eds.), Handbook of neuropsychology (Vol. 8, pp. 63-131). Amsterdam: Elsevier.

Roediger, H. L., III, Weldon, M. S., \& Challis, B. H. (1989). Explaining dissociations between implicit and explicit measures of retention: A processing account. In H. L. Roediger III \& F. I. M. Craik (Eds.), Varieties of memory and consciousness: Essays in honor of Endel Tulving (pp. 3-41). Hillsdale, NJ: Erlbaum. 
SCHACTER, D. L. (1987). Implicit memory: History and current status. Journal of Experimental Psychology: Learning, Memory, \& Cognition, 13, 501-518.

SCHACTER, D. L. (1992). Priming and multiple memory systems: Perceptual mechanisms of implicit memory. Journal of Cognitive Neuroscience, 4, 244-256.

ShePARD, R. N. (1980). Multidimensional scaling, tree-fitting, and clustering. Science, 210, 390-398.

ShePARD, R. N. (1987). Toward a universal law of generalization for psychological science. Science, 237, 1317-1323.

Shimamura, A. P., \& Squire, L. R. (1984). Paired-associate learning and priming effects in amnesia: A neuropsychological study. Journal of Experimental Psychology: General, 113, 556-570.

Thompson-Schill, S. L., \& Gabrieli, J. D. E. (1999). Priming of visual and functional knowledge on a semantic classification task. Journal of Experimental Psychology: Learning, Memory, \& Cognition, 25, 41-53.

Tulving, E. (1979). Relation between encoding specificity and levels of processing. In L. S. Cermak \& F. I. M. Craik (Eds.), Levels of processing in human memory (pp. 405-428). Hillsdale, NJ: Erlbaum.

Tulving,E., \& Thomson, D. M. (1973).Encoding specificity and retrieval processes in episodic memory. Psychological Review, 80, 359-380.

Tzeng, O. C. S. (1975). Reliability and validity of semantic differential E-P-A markers for an American English representative sample. Psychological Reports, 37, 292.

TzenG, O. C., \& MAY, W. H. (1975). More than E, P, and A in semantic differential scales: An answer to questions raised by T. M. Lane. International Journal of Psychology, 10, 101-117.

VAIDYA, C. J., \& GABRIELI, J. D. E. (2000). Picture superiority in conceptual memory: Dissociative effects of encoding and retrieval tasks. Memory \& Cognition, 28, 1165-1172.

Vaidya, C. J., Gabrieli, J. D. E., Keane, M. M., Monti, L. A., GutierreZ-Rivas, H., \& Zarella, M. M. (1997). Evidence for multiple mechanisms of conceptual priming on implicit memory tests. Journal of Experimental Psychology: Learning, Memory, \& Cognition, 23, 1324-1343.

Vriezen, E. R., Moscovitch, M., \& Bellos, S. A. (1995). Priming ef- fects in semantic classification tasks. Journal of Experimental Psychology: Learning, Memory, \& Cognition, 21, 933-946.

Wickens, D. D., \& LindBeRG, M. A. (1975). A test of four proposed new dimensions of semantic space. Bulletin of the Psychonomic Society, 6, 381-382.

Witherspoon, D., \& Moscovitch, M. (1989). Stochastic independence between two implicit memory tasks. Journal of Experimental Psychology: Learning, Memory, \& Cognition, 15, 22-30.

\section{NOTES}

1. The results of Osgood et al., 1957, Analysis I were replicated with 20 undergraduate students from Vanderbilt University. Thirty semantic scales was selected from the original Analysis I, including the 6 scales used in the present study. The subjects rated 200 nouns with these scales. Factor analysis was then performed on the cross-correlation between scales. Loadings of the 30 scales on the first three factors extracted were compared with those of Analysis I. For the first factor, the loadings of the scales had a correlation of .97 with their loadings on the first factor in Analysis I; for the second and third factors, the correlations were .80 and .83 , respectively (all $p \mathrm{~s}<.001$ ).

2. Parametric tests between the minimal, the intermediate, and the maximal distance groups were not applicable because some data in different groups were from the same subjects.

3. The correlation between consistency rate and semantic distance could also be the result of bias on response type from the study task, as a form of repetition priming. It was possible that the more closely related the scales, the greater the bias toward consistent responding. A post hoc item analysis indicated that this was unlikely. There was a slight tendency for old test items in the intermediate distance conditions to receive whatever hand response they had received during study, as compared with the type of response they would otherwise have received if they had been new items. Yet exactly the same result was found for old items in the maximal distance conditions also. It is very unlikely that biased responses were behind the correlation between consistency rate and semantic distance. 
APPENDIX

Complete Experimental Item List

\begin{tabular}{|c|c|c|c|c|}
\hline action & crowd & gossip & mind & scene \\
\hline angel & cry & ground & money & school \\
\hline answer & custom & information & moon & science \\
\hline apprentice & danger & group & mother & sense \\
\hline army & daughter & health & mouth & silver \\
\hline art & decision & heat & movement & situation \\
\hline artist & difference & hill & music & skull \\
\hline attention & difficulty & history & nature & sleep \\
\hline baby & discovery & home & newspaper & sorrow \\
\hline bagel & distance & honey & night & star \\
\hline ball & doctor & hospital & oil & steel \\
\hline bank & cream & house & paper & stone \\
\hline battle & dollar & hurt & party & story \\
\hline beauty & door & illness & picture & stream \\
\hline bed & doubt & itch & piece & summer \\
\hline bird & dream & job & plan & surface \\
\hline blood & duty & joy & poet & sweat \\
\hline boat & ear & judge & police & thought \\
\hline book & effort & kiss & politics & town \\
\hline box & egg & knowledge & power & tree \\
\hline bread & empire & lake & presence & trouble \\
\hline bridge & end & laugh & pressure & victory \\
\hline car & evening & law & price & wall \\
\hline chance & example & leak & problem & water \\
\hline chief & eye & left & promise & wife \\
\hline church & face & life & question & wind \\
\hline city & factory & light & relation & window \\
\hline cloud & failure & limit & report & winter \\
\hline college & farm & loss & result & wish \\
\hline color & fear & love & river & woman \\
\hline committee & fire & machine & road & world \\
\hline connection & flower & $\operatorname{man}$ & room & \\
\hline cost & football & meeting & rule & \\
\hline country & force & middle & sailor & \\
\hline court & game & milk & sand & \\
\hline
\end{tabular}

(Manuscript received November 10, 2002;

revision accepted for publication May 30, 2003.) 